\title{
Gamificação como Estratégia no Engajamento de Estudantes do Ensino Fundamental
}

\author{
Luma da Rocha Seixas ${ }^{1,2}$, Alex Sandro Gomes ${ }^{1,2}$, Ivanildo J. Melo Filho ${ }^{1,2,3}$, \\ Rodrigo Lins Rodrigues ${ }^{1,2}$ \\ ${ }^{1}$ Centro de Informática - Universidade Federal de Pernambuco (UFPE) \\ ${ }^{2}$ Grupo de Pesquisa Ciências Cognitivas e Tecnologia Educacional (CCTE) \\ Caixa Postal 7.851 - 54.740-540 - Recife - PE - Brazil \\ ${ }^{3}$ Instituto Federal de Pernambuco - Campus Belo Jardim (IFPE)
}

Av. Sebastião Rodrigues da Costa, S/N - CEP: 55155-730 - Belo Jardim - PE - Brazil

\begin{abstract}
This research aims to evaluate efectiveness the usage of gamification as strategy on student engagement in the $9^{\text {th }}$ year of elementary school. For this, we realized a field research with 61 students from a public school. Were used two badging platforms - ClassDojo and ClassBadges - to evaluate the gamification effects. At data collected we used techniques as: observation, semi-structured interview and questionnaries. The results were done statistically through multivariated technique know as cluster analysis, this allowed to classify the students in 4 groups. Therefore, the results showed that the students getting better averages were those who receveid more rewards from teacher.
\end{abstract}

Resumo. Esta pesquisa teve como objetivo avaliar a efetividade da utilização de Gamificação como estratégia sobre o engajamento de alunos do $9^{\circ}$ ano do ensino fundamental. Para tanto, foi realizado um estudo de campo com 61 alunos de uma escola pública. Foram utilizadas duas plataformas de recompensa-ClassDojo e ClassBadges - no intuito de analisar os efeitos da gamificação. Para a coleta de dados utilizou-se técnicas como: observação, entrevista semiestruturada e questionário. Os resultados foram tratados estatisticamente pela técnica multivariada conhecida como Análise de Conglomerados (Cluster). Foi possivel classificar os alunos em 4 grupos e concluir que os alunos que obtiveram as melhores médias nos indicadores foram os que receberam mais recompensas do professor.

\section{Introdução}

As novas gerações fazem uso amplo de tecnologias como o computador, celulares e os consoles de videogame (LAZZARO, 2005; McGONIGAL, 2011). Prensky (2002) adota o conceito de "nativos digitais" para se referir à geração de indivíduos que cresce em meio a esses hábitos. Para o autor, os "nativos digitais" não se preocupam com a leitura de manuais de instrução nem recorrem a técnicos especializados, uma vez que "falam" a linguagem digital desde que nasceram (PRENSKY, 2002). Eles preferem descobrir por si o funcionamento da tecnologia que tem em mãos.

Pesquisas sugerem o uso de jogos como uma tecnologia especializada e com alto sucesso em engajar e motivar seus usuários, fazendo com que estes permaneçam em uma tarefa por um longo período de tempo (McGONIGAL, 2011; Lazzaro (2005) identificou que as pessoas jogam não exclusivamente pela atividade do jogo em si, mas 
pelas experiências que ele proporciona. Dentre elas, destacam-se: a adrenalina, a aventura, o desafio mental e pela possibilidade de estar em uma atividade divertida na qual é possível estar sozinho ou na companhia de amigos.

Diante desses fatos, é crescente o interesse pelo aproveitamento dessas experiências na área de educação (LEE \& HAMMER, 2011). Por um lado, alguns pesquisadores focam no potencial dos jogos para funcionar como ferramentas educacionais inovadoras. Investigações tem evidenciado resultados positivos com relação à experiência de aprendizagem (JOHNSON, 2005). Outras, relatam melhorias significativas da motivação. E, consequentemente, do engajamento (BARNES et al., 2008). Por outro lado, alguns estudos não se concentram em utilizar diretamente os jogos na educação, mas apenas em alguns aspectos, tais como: medalhas, pontos, rankings e feedback (LEE \& HAMMER, 2011; DOMÍNGUEZ et al., 2013). Essa possibilidade de favorecer a motivação e engajamento tem sido explorada através de uma técnica chamada de gamificação (ZICHERMAN \& CUNNINGHAM, 2011; KAPP, 2012). Esta técnica envolve o uso de elementos de game-design e características dos jogos em contextos não-game (DETERDING et al., 2011).

Assim, este trabalho tem como objetivo avaliar a efetividade da gamificação como estratégia no engajamento de estudantes do ensino fundamental. Utilizou-se duas plataformas web para realizar a mecânica de recompensar atividades e comportamentos realizados pelos alunos: ClassDojo e ClassBadges.

O artigo está organizado da seguinte forma: A seção 2 traz uma discussão sobre os indicadores de engajamento, a seção 3 evidencia a gamificação aplicada à educação. A seção 4 apresenta o método utilizado no desenvolvimento deste trabalho. Na sequência os resultados obtidos são discutidos na seção 5. E, por fim, a seção 6 apresenta as considerações finais.

\section{Indicadores de Engajamento dos Alunos}

Uma série de estudos sobre o engajamento de alunos foram encontrados na literatura. Contudo, ainda não existe consenso sobre sua definição (FREDRICKS et al. 2004; GIBBS \& POSKITT, 2010; LIBBEY, 2004). Dentre o universo investigado, os conceitos encontrados geralmente incluem um componentes psicológico e comportamental como apresentado em Beer et al. (2010) e Fredricks et al. (2004). Outros autores, a exemplo de Sullivan et al. (2009) e Fredricks et al. (2004), analisam o engajamento com o intuito de discutir as atitudes dos alunos em relação à escola. Para os autores, o não-engajamento do aluno geralmente indica o abandono da mesma. Encontra-se na literatura, o conceito também relacionado à forma como os estudantes interagem com as atividades escolares que lhes são propostas. Quando eles estão envolvidos com suas atividades, tendem a persistir na realização destas, apesar dos desafios e obstáculos (SITWAT \& DAVID, 2012).

De acordo com Fredricks, Blumenfeld e Paris (2004), podem ser distinguidas na literatura três definições para o engajamento: engajamento comportamental, engajamento emocional e engajamento cognitivo. $O$ engajamento comportamental envolve a participação e o envolvimento dos estudantes em atividades escolares e em atividades extracurriculares e as condutas positivas empreendidas pelos estudantes durante a resolução das atividades. O engajamento emocional envolve as reações afetivas e emocionais dos estudantes diante das atividades, dos sujeitos e de outros 
elementos que compõe o ambiente escolar. Interesse, felicidade, bem estar, desgosto, ansiedade, frustração são exemplos de tais reações. $\mathrm{O}$ engajamento cognitivo envolve o investimento psicológico do estudante na aprendizagem. Ele é marcado pelo esforço empreendido pelo estudante para compreender o que é estudado e para atingir níveis mais elevados de compreensão sobre determinado tópico de estudo.

Nesse sentido, esse trabalho buscou inicialmente identificar na literatura quais indicadores são pontados nos estudos como resultado ou processo que contribui para o engajamento de estudantes. Como resultado, obteve-se inicialmente 10 indicadores: autonomia, execução, social, entrega, participação, colaboração, cooperação, questionamento, organização do ambiente e diversão.

\section{Gamificação Aplicada à Educação}

Gamificação - do inglês, Gamification - refere-se ao conjunto de técnicas que incorpora elementos de jogos em contextos cotidianos. Tem por objetivo oferecer oportunidades para auxiliar escolas a minimizar problemas de motivação e engajamento dos alunos (LEE e HAMMER, 2011). Para estes, com a gamificação não se pretende ensinar com jogos ou através de jogos mas sim usar mecânicas de jogos como forma de promover a motivação e o engajamento dos alunos. Nesta direção, Domínguez et al. (2013) apontam que o principal objetivo da gamificação quando aplicada à educação é utilizar mecânicas de jogos que os tornam interessantes na concepção de iniciativas educativas, como também, de seus conteúdos na tentativa de torná-los mais atrativos.

Na perspectiva de Simões et al. (2012) a abordagem de gamification aumenta o nível de engajamento dos alunos sem o uso de um jogo específico. Esta abordagem, tem por objetivo extrair os elementos que os tornam agradáveis e divertidos e os adapta para serem utilizados nos processos de ensino. Segundo Deterding et al. (2011a), o termo Gamification se originou na área de mídia digital por volta do ano de 2008. Contudo, segundo os autores, este passou a popularizar-se apenas no segundo semestre de 2010.

Segundo Kapp (2012), quando bem utilizadas, as mecânicas podem deixar os alunos mais engajados. O autor parte do princípio que todo educador realiza uma espécie de "jogo" com seus alunos, propondo desafios e oferecendo soluções. Contudo, ele alerta que o uso de gamificação na educação não se resume a dar pontos toda vez que um aluno visualiza uma lição ou entrega uma tarefa.

\section{Método}

Foi adotada abordagem qualitativa e quantitativa para analisar a efetividade do uso de mecânicas de gamificação sobre o engajamento de alunos no ensino fundamental. Nas subseções que seguem, são apresentado os participantes, a disciplina objeto de estudo dos participantes, as plataformas utilizadas, as estratégias de gamificação adaptadas e os procedimentos.

\subsection{Contexto da Pesquisa e Participantes}

Para a realização da pesquisa, foi escolhido o Colégio de Aplicação, escola pública federal de ensino fundamental da cidade de Recife/PE. Esta instituição de ensino é vinculada e mantida pela UFPE - Universidade Federal de Pernambuco. Participaram do estudo 61 alunos de duas turmas do $9^{\circ}$ ano do Ensino Fundamental de ambos os sexos, com faixa etária entre 13 e 14 anos de idade. Nesse estudo, os alunos serão 
identificados segundo a nomenclatura "Aln $\mathbf{X X}$ “, onde: Aln - representa o aluno e $\mathbf{X X}$ - sequencial de identificação dos alunos.

Foi aplicado um questionário, descrito na seção 4.6, para qualificar o perfil dos estudantes. De um total de 61 alunos, 48 responderam ao questionário disponibilizado. Ao avaliar o perfil estudantes, identificou-se $95,8 \%$ possuem computador, e destes, $89,5 \%$ utilizam essa ferramenta para estudar. A maioria destes fica em média até 4 horas acessando a internet, especificamente os seguintes conteúdos: redes sociais (Facebook, Instagram, Twitter, entre outros), Sites de Entretenimento (Youtube, blogs de humor, entre outros) e Conteúdos educativos (que auxiliem as tarefas escolares), em ordem de preferência. O professor participante possui graduação em Licenciatura em Desenho e Plástica pela Universidade Federal de Pernambuco(UFPE) e mestrado em Educação Matemática e Tecnológica pela mesma instituição.

\subsection{Procedimentos}

\subsection{1 - Fase 1: Seleção e Entendimento dos Objetivos da Disciplina}

Com o intuito de identificar quais atividades seriam gamificadas e de qual forma, procurou-se inicialmente identificar e entender as especificações do plano de ensino da disciplina disponibilizado pelo professor. Foi escolhida a disciplina 'Desenho Geométrico'. O plano de ensino é definido com 4 objetivos principais. Tais objetivos Apresentados na Tabela 1 - são pautados no projeto político pedagógico da instituição e buscaram atender às necessidades do professor. Os objetivos descritos são:

Tabela 1. Objetivos da disciplina.

\begin{tabular}{|l|l|}
\hline \multicolumn{1}{|c|}{ Objetivos } & \multicolumn{1}{|c|}{ Descrição } \\
\hline $\begin{array}{l}\text { Objetivos } \\
\text { Atitudinais }\end{array}$ & $\begin{array}{l}\text { São os tipos mais complexos de objetivos. Uma vez que eles estão relacionados ao } \\
\text { "ser". Está relacionado ao conjunto de valores, atitudes que fazem parte da } \\
\text { personalidade do aluno. Esse item engloba situações como: participação na sala de } \\
\text { aula, trabalho em equipe, entrega das atividades no prazo, curiosidade, vontade de } \\
\text { aprender, entre outros. }\end{array}$ \\
\hline $\begin{array}{l}\text { Objetivos } \\
\text { Cognitivos }\end{array}$ & $\begin{array}{l}\text { Fornecem uma meta na qual um aluno ou grupo de alunos está trabalhando - tomada } \\
\text { de decisões, avaliação, pensamento ou resolução de problemas. Um objetivo } \\
\text { cognitivo determina qual a tarefa específica a ser realizada e como ela irá ajudar os } \\
\text { alunos a desenvolverem a aprendizagem cognitiva. No caso da disciplina, estes } \\
\text { estavam ligados ao pensamento geométrico. }\end{array}$ \\
\hline $\begin{array}{l}\text { Objetivos } \\
\text { Conceituais }\end{array}$ & $\begin{array}{l}\text { Estão associados às "matérias" ou "conteúdos", ou seja, elementos específicos dentro } \\
\text { do saber daquela disciplina, como: triângulos, quadriláteros, trapézios e etc. }\end{array}$ \\
\hline $\begin{array}{l}\text { Objetivos } \\
\text { Instrumentais }\end{array}$ & $\begin{array}{l}\text { Dizem respeito a correta utilização dos instrumentos de desenho técnico (compasso, } \\
\text { esquadros e lapiseira), a qualidade do desenho, além da limpeza e organização. }\end{array}$ \\
\hline
\end{tabular}

\subsection{2 - Fase 2: Identificação das Ferramentas de Comunicação Utilizada}

No intuito de ampliar a sua comunicação com os alunos o professor utilizava a ferramenta de Grupos do Facebook. No ambiente, eram postadas atividades referentes à disciplina e os alunos também poderiam tirar suas dúvidas com relação ao conteúdo. $\mathrm{Na}$ ferramenta, o professor solicitou aos alunos que se cadastrassem nas plataformas a serem utilizadas. Foi informado que, ao ajudar um colega, por exemplo, os alunos pudessem identificar-se, tanto no Facebook quanto na sala para receber um "dojo". 


\subsection{3 - Fase 3: Seleção das Plataformas online de Recompensa: ClassDojo e ClassBadges}

O ClassDojo ${ }^{\boldsymbol{I}}$ é uma plataforma gratuita e online cuja proposta é recompensar o comportamento de estudantes. Esta, busca incentivar comportamentos positivos específicos como persistência, curiosidade e trabalho em equipe. A proposta é construir a outra metade da educação, que vai além das boas notas nas provas, e sim incentivar a construção de um bom caráter nos alunos. O ClassBadges é uma ferramenta gratuita por meio da qual os professores podem premiar as habilidades de seus alunos e/ou domínio acadêmico. O professor pode personalizar os emblemas para sua sala de aula ou escola. O ClassBadges foi escolhido por além de ser uma ferramenta gratuita, possui uma curva de aprendizado baixa e, possui a facilidade de alinhar a criação dos badges com objetivos acadêmicos existentes.

\subsection{4 - Fase 4: Adequação das Estratégias de Gamificação}

O ClassDojo foi utilizado para recompensar os objetivos atitudinais. Na ferramenta há a possibilidade de criação de comportamentos positivos e outros que precisam ser melhorados. Esta então gera um relatório com todas as interações que acontecem, o que facilita o trabalho do professor quando este precisar avaliar os alunos individualmente. O serviço permite que os professores atribuam aos alunos recompensas em tempo real a partir da análise do comportamento em sala de aula. O ClassBadges teve como propósito acompanhar os objetivos instrumentais, cognitivos e conceituais. Nele, o professor personalizou os emblemas para sua sala de aula. Uma das grandes vantagens da ferramenta foi a facilidade em alinhar os objetivos acadêmicos com as medalhas criadas. Com a definição das atividades a serem realizadas e das estratégias de gamificação escolhidas, deu-se início a utilização das plataformas.

A ferramenta ClassDojo disponibiliza uma opção para "Personalizar Comportamentos". Nesta opção, o docente tem acesso a uma interface no qual pode escolher ícones e associá-los a uma descrição com o intuito de atribuir comportamentos positivos, na Figura 1 e aqueles que precisam ser melhorados, Figura 2.

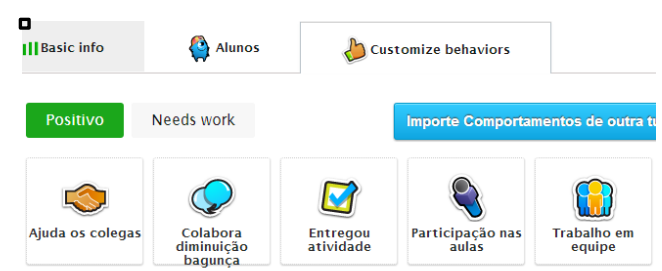

Figura 1. Comportamentos Positivos no ClassDojo.

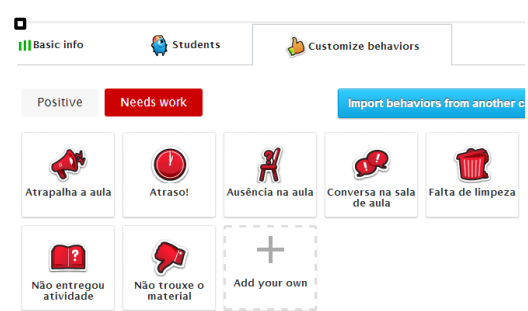

Figura 1. Comportamentos que precisam ser melhorados no ClassDojo.

Da mesma forma que ocorreu com a ferramenta anterior, os alunos foram também cadastrados no ClassBadges pelo professor, que também esteve responsável pela criação das "medalhas" que cada aluno iria receber. O profesor optou por colocar nomes bastante sugestivos, resgatando analogias com jogos de vídeo games que são comuns no dia-a-da dos alunos. Foram definidas as medalhas, onde cada uma delas apresenta um nome e uma descrição que é vista pelo aluno no momento que ele a

\footnotetext{
${ }^{1}$ ClassDojo - Disponível na Internet: <http://www.classdojo.com>. Acesso em: 3 mai. 2014.
} 
recebe, identificando uma habilidade que o mesmo adquiriu. O Quadro 2 apresenta alguns exemplos dessas.

Quadro 1. Exemplos de medalhas do ClassBadges.

\begin{tabular}{|l|l|l|}
\hline Badge & Título & Mensagem \\
\hline $\begin{array}{l}\text { Senhor da } \\
\text { Caligrafia }\end{array}$ & Parabéns! Você domina a arte lendária dos antigos sábios: a caligrafia. \\
\hline $\begin{array}{l}\text { Senhor dos } \\
\text { Traçados }\end{array}$ & $\begin{array}{l}\text { Nossa! Você sabe manusear as armas sagradas de Desenho (Esquadros, } \\
\text { Compasso, etc.). Sabe diferenciar o traçado, como também apresenta seu } \\
\text { trabalho com organização e limpeza. }\end{array}$ \\
\hline $\begin{array}{l}\text { Pensamento } \\
\text { Seométrico }\end{array}$ & $\begin{array}{l}\text { Você atingiu o mais alto grau, indo além dos outros sentidos. Apresenta bom } \\
\text { raciocínio abstrato e geométrico e consegue fazer relações entre conteúdos }\end{array}$ \\
\hline
\end{tabular}

Após a seleção, adequação e preparação plataformas e no decorrer das aulas os alunos foram recebendo os "dojos". Esta atribuição foi fornecida para as interações que ocorriam na ferramenta ClassDojo. As interações na ferramenta ClassBadges ocorreram apenas ao término da disciplina. Esta ação permitiu ao professor avaliar as habilidades dos alunos com relação a disciplina e premiá-las com as medalhas ou badges.

\subsection{Coleta de dados}

Esta pesquisa adotou um paradigma misto, com técnicas exploratórias de natureza qualitativa complementadas com técnicas quantitativas. Assim, na direção de investigar a gamificação como estratégia no engajamento dos estudantes, foram utilizadas diferentes técnicas de coleta de dados como observação, entrevista semiestruturada e questionário.

Para a análise dos dados obtidos optou se por realizar a Análise de Clustesr. Essa análise, também conhecida como Análise de conglomerados, compreende um grupo de técnicas multivariadas cuja finalidade primária é agregar objetos com base nas características que eles possuem (HAIR et al., 2005). A análise de cluster classifica objetos de modo que cada objeto é muito semelhante aos outros no agrupamento em relação a algum critério de seleção pré-determinado (HAIR et al., 2005). A ferramenta utilizada para a análise foi "Software $R$ ", versão 3.0.1 para Windows. A Análise de Clusters foi aplicada na análise dos dados obtidos através do questionário, com o objetivo de classificar os alunos utilizando os indicadores de engajamento como base e dessa forma melhor identificar os efeitos da gamificação.

\section{Resultados e Discussão}

Esta seção discute os dados coletados nesta pesquisa, que possibilitaram comprovar a efetividade do uso de gamificação como estratégia no engajamento de estudantes do ensino fundamental. Foram ainda identificados, a partir da revisão da literatura, indicadores de engajamento que foram utilizados no intuito de avaliar $o$ comprometimento dos alunos.

O engajamento dos alunos - independente da forma como é promovido - deve ser realizado com base nos objetivos educacionais (BULGER et al., 2008; BEER et al., 
2010). É necessário considerar ainda o perfil dos alunos e a infraestrutura disponível na escola (PORTER, 2006; LANASA et al., 2009; LAIRD et al., 2009). Ficou evidenciado que alinhar os objetivos atitudinais, cognitivos, conceituais e instrumentais é de fundamental importância para o engajamento dos estudantes. Identificar quais comportamentos devem ser premiados ou de certa forma, advertidos ajuda o professor a qualificar os seus alunos, e também beneficia aos alunos, suas estes passam a ter consciência de atitudes em sala de aula, como: o que pode ser melhorado e quais habilidades está adquirindo.

No que diz respeito à utilização das plataformas ClassDojo e ClassBadges, estas se apresentaram como uma forma divertida de apresentar o desempenho dos alunos. No classdojo, estes eram avaliados a partir de suas interações com os demais na sala de aula, sua participação e empenho em realizar suas atividades. O ClassBadges premiava as habilidades cognitivas adquiridas pelos alunos. Notou-se que o professor teve um papel decisivo no engajamento dos alunos (SAGAYADEVAN e JEYARAJ, 2012). Este, desde o início da pesquisa mostrou-se prestativo e empenhado em utilizar as ferramentas com os alunos. Sua habilidade com o uso de tecnologia, também foi um fator positivo na execução das atividades. Com relação aos alunos, estes mostraram curiosidade desde o início da pesquisa com relação as ferramentas. Foi observado ainda que, ocasionalmente, eles mesmos cobraram do professor as "medalhas". Observou-se também uma motivação maior em ajudar aos colegas no intuito de receber a medalha correspondente ao comportamento de contribuir com os colegas.

\subsection{Recompensas por desempenho}

Os indícios iniciais apontam que os alunos mostraram-se comprometidos com a realização de suas atividades. Contudo, estes queriam ser reconhecidos por isso. Conforme comentário realizado por um dos alunos, indicando que este ajudou um dos colegas, em uma rede social. O Aln01 disse em momentos distintos: "Eu ajudei Aln02 e Aln03 nas atividades ". "Se você ajudou ele (o professor) disse q podia postar aki q ele dava ponto no ClassDojo :)".

No que se refere à utilização das ferramentas, os estudantes puderam perceber suas habilidades através das medalhas do ClassBadges e com utilização do ClassDojo, puderam ter reconhecidas algumas atividades que muitas vezes passam despercebidas pelo professor, mas que são importantes ao longo do acompanhamento do aluno. As duas ferramentas proporcionaram um repositório com informações importantes sobre os comportamentos e habilidades, tanto para o aluno identificar suas ações em sala de aula $\mathrm{e}$, dessa forma poder melhorar suas atitudes, quanto para o professor. As ferramentas tornaram-se uma espécie de diário do comportamento e das habilidades dos alunos, ao responder na entrevista, sobre a sua experiência com as plataformas, o professor relatou: "Acredito que as duas ferramentas ajuda a avaliarmos como o nosso plano de ensino está funcionando na prática, pois se os objetivos do plano estão traduzidos nos badges, mas não estão sendo pontuados isso pode apontar uma disparidade entre a prática e o planejamento”.

\subsection{Efetividade das mecânicas}

Resultados relevantes foram obtidos com a utilização das ferramentas. Foi possível observar, tanto no contexto da sala de aula, quanto em uma rede social utilizada pelos alunos, o Facebook. Na sala de aula, os próprios alunos cobravam do professor a 
realização de atividades e queriam receber seus “dojos'. Além disso, A partir de uma das conversas com o professor, durante a observação este relatou que o fato de ter os pais cadastrados cria um certo 'receio' nos alunos. Com isso, ao demonstrar interesse em colocar 'dojos negativos' o professor observava que inibia atitudes negativas dos alunos. As análises preliminares dos dados estão consoantes com Hakulinen et al. (2013), sobre a utilização gamificação, mais especificamente das medalhas podem contribuir para o engajamento dos alunos. Através da análise de cluster, foi possível classificar os alunos em 4 grupos. A Tabela 2 apresenta a distribuição da amostra segundo os quatro clusters obtidos, numa tentativa de definir perfis em função de um conjunto de indicadores já referidos, podendo-se constatar que o grupo que integrou mais estudantes foi o cluster $2(33,3 \%)$.

Tabela 2. Clusters identificados.

\begin{tabular}{|l|c|c|}
\hline \multicolumn{2}{|c|}{ Distribuição dos estudantes nos quatro Clusters identificados } \\
\hline \multicolumn{1}{|c|}{ Clusters } & N & \% \\
\hline Cluster 1 & 8 & 16,6 \\
\hline Cluster 2 & 16 & 33,3 \\
\hline Cluster 3 & 11 & 22,9 \\
\hline Cluster 4 & 13 & 27,0 \\
\hline
\end{tabular}

A comparação entre os grupos mostrou que o grupo 4, foi o que atingiu as maiores médias entre os indicadores de engajamento, dos 11 apresentados em 8 a média foi superior aos demais (autonomia, execução, social, entrega, participação, colaboração, trabalho em equipe e questionar). Por outro lado, o grupo 1 foi o que obteve as menores médias. Dos 11 indicadores de engajamento, obteve as 6 médias mais baixas (autonomia, participação, colaboração, organização do ambiente, zelo e diversão). A Figura 3, apresenta um gráfico com as médias obtidas pelos grupos em todos os indicadores de engajamento.

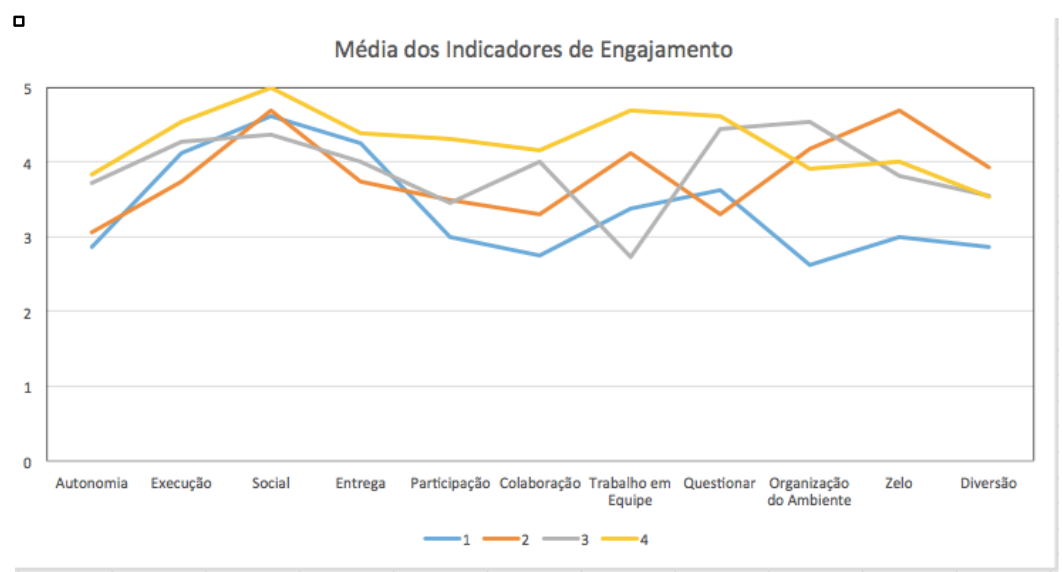

Figura 2. Média dos indicadores.

Os resultados mostram que o cluster 1 era formado por alunos que apresentaram os valores menos significativos, ou seja, os alunos estavam menos comprometidos. Os clusters 2 e o 3 apresentaram resultados muito similares nos indicadores, nesse caso, os alunos demonstravam interesse contudo isso ocorria ocasionalmente, já os alunos do 4 demonstraram um maior índice de engajamento em quase todos os. Essas informações, foram contrastadas com o número de badges recebidos por cada grupo, e observou-se que os grupos que obtiveram as melhores médias receberam mais badges do professor. 


\section{Considerações finais}

Este trabalho analisou a efetividade de mecânicas de gamificação para estimular o engajamento entre estudantes do ensino fundamental. A investigação mostrou que o uso de gamificação teve efeitos positivos no engajamento dos alunos. Os alunos que apresentaram os maiores índices de engajamento nos indicadores, também foram os que receberam mais badges do professor, em contraste, ficaram os alunos que apontaram menores índices nos indicadores e que receberam menos badges do professor. É importante observar que, esse processo de construção de estratégias de gamificação deve sempre estar alinhado aos objetivos educacionais. Nessa pesquisa, o fato de seguirmos o plano de curso da disciplina foi muito importante, tanto para o professor que conseguiu acompanhar melhor os alunos, e não precisou modificar muito a sua didática na sala de aula, quanto para os alunos, que acompanharam melhor o seu desenvolvimento.

Uma contribuição que pode ser citada é a constatação da relevância e benefícios resultante da gamificação como estratégia no engajamento. Visto que essa possibilidade, entre tantos aspectos, proporciona ao professor: Uma visão geral do comportamento dos seus alunos, em termos de comportamento na sala de aula e habilidades cognitivas adquiridas. Além disso, permite aos alunos ter reconhecidas quais competências alcançou e com isso, avaliar quais precisa adquirir.

Destaca-se ainda, que o professor teve um papel fundamental na realização do estudo. Desde o início, mostrou-se disponível e foi praticamente autônomo na utilização das ferramentas. Este, estimulava os alunos para a realização das atividades no intuito de receber as medalhas e acompanhava o desempenho dos alunos atribuindo as habilidades que haviam adquirido. À exceção da observação da sala de aula e conversas com o professor no antes ou final das aulas, não houveram intervenções do pesquisador.

\section{Agradecimentos}

Luma Rocha Seixas foi bolsista CNPQ de mestrado da UFPE - Centro de Informática, orientada pelo Prof. Dr. Alex Sandro Gomes, bolsista DT Nível 2 pelo CNPq apoiado pelos processos $n^{\circ} 310466 / 2012-1$ e n ${ }^{\circ} 475634 / 2013-6$.

\section{Referências}

Akey, T. M. School context, student attitudes and behavior, and academic achievement: An exploratory analysis. 2006. New York: MDRC.

Barnes, T.; Powell, E.; Chaffin, A.; Lipford, H. Game2Learn: improving the motivation of CS1 students. In Proceedings of the 3rd International Conference on Game Development in Computer Science Education (GDCSE '08). 2008. ACM, New York, NY, USA, 1-5.

Beer C.; Clark K.; Jones D. (2010) Indicators of engagement. In Curriculum, technology and transformation for an unknown future. Proceedings of ASCILITE Sydney 2010.

Bulger, M. E., Mayer, R. E., Almeroth, K. C., \& Blau, S. D. (2008). Measuring Learner Engagement in Computer-Equipped College Classrooms. Journal of Educational Multimedia and Hypermedia, 17(2), 129-143.

Deterding, S; Dixon, D.; Khaled, R.; Nacke, L. From Game Design Elements to Gamefulness: Defining “Gamification”. MindTrek'11.2011. 
Domínguez, A.; Saenz-de-Navarrete, J.; de-Marcos, L.; Fernández-Sanz, L.; Pagés, C.; Martínez-Herráiz, J.Gamifying learning experiences: Practical implications and outcomes, Computers \& Education, Volume 63, April 2013, Pages 380-392,

Fredricks, J. A.; Blumenfeld P. C.; Paris A. H.School Engagement: Potential of the Concept, State of the Evidence. Review of Educational Research. Spring 2004, Vol. 74, No. 1, pp. 59-109.

Gibbs R.; Poskitt J. Student Engagement in the Middle Years of Schooling (Years 710): A Literature Review. 2010. Report to the Ministry of Education.

Hair, J. F.; Anderson, R. E.; Tatham, R. L.; Black, W. C. Análise Multivariada de

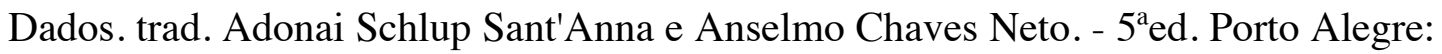
Bookman, 2005.

Hakulinen, L.; Auvinen, T.; Korhonen, A., Empirical Study on the Effect of Achievement Badges in TRAKLA2 Online Learning Environment. Learning and Teaching in Computing and Engineering (LaTiCE), 2013.

Johnson, W. L. Lessons learned from games for education. In ACM SIGGRAPH 2005 Educators program (SIGGRAPH '05), Patricia Beckmann-Wells (Ed.). ACM, New York, NY, USA, , Article 31.

Kapp, K. The Gamification of Learning and Instruction: Game-Based Methods and Strategies for Training and Education. 2012. San Francisco: Pfeiffer. ISBN: 978-1118-09634-5.

LaNasa, S.M., Cabrera, A.F., \& Trangsrud, H. (2009). The construct validity of student engagement: A confirmatory factor analysis approach. Research in Higher Education, 50, 315-332.

Lazzaro, N. Why We Play Games: Four Keys to More Emotion Without Story. Technical Report, XEODesign, Inc (2005).

Lee, J. J. ; Hammer, J. Gamification in Education: What, How, Why Bother? . 2011.

Libbey, H. P. Measuring Student Relationships to School: Attachment, Bonding, Connectedness, and Engagement. Journal of School Health. 2004. 74: 274-283

McGonigal, J. Reality Is Broken: Why Games Make Us Better and How They Can Change the World. Penguin, London, 2011.

Prensky, M. The motivation of gameplay: The real twenty-first century learning revolution. On the Horizon, Vol. 10. (2002) Iss: 1, pp.5 - 11.

Sagayadevan, V; Jeyaraj, S. The role of emotional engagement in lecturer-student interaction and the impact on academic outcomes of student achievement and learning. Journal of the Scholarship of Teaching and Learning, Vol. 12, No. 3, September 2012, pp. 1 - 30.

Sullivan P.; Mornane, A.; Prain, V.; Campbell, C.; Deed, C.; Drane, S.; Faulkner, M.; McDonough A.; Smith, C. (2009) Junior Secondary Students' Perceptions of Influences on Their Engagement with Schooling. Australian Journal of Education. 2009.

Zepke N.; Leach L.; Butler P. Student Engagement: What Is It and What Influences It?. Teaching \& Learning Research Initiative. 2010.

Zichermann, G. ; Cunningham, C. Gamification by Design: Implementing Game Mechanics in Web and Mobile Apps. O’Reilly, Sebastopol, 2011. 